\title{
Villus Tip
}

National Cancer Institute

\section{Source}

National Cancer Institute. Villus Tip. NCI Thesaurus. Code C83469.

The distal end of a villus. 